\title{
BOUNDED TYPE INTERVAL EXCHANGE MAPS
}

\author{
DONG HAN KIM AND STEFANO MARMI
}

\begin{abstract}
Irrational numbers of bounded type have several equivalent characterizations. They have bounded partial quotients in terms of arithmetic characterization and in the dynamics of the circle rotation, the rescaled recurrence time to $r$-ball of the initial point is bounded below. In this paper, we consider how the bounded type condition of irrational is generalized into interval exchange maps.
\end{abstract}

\section{INTRODUCTION}

An irrational $\theta$ is said to be of bounded type (or constant type) if its partial quotients $\left(a_{k}\right)$ of the continued fraction expansion are bounded. This condition is equivalent that there exists a constant $c>0$ such that

$$
\liminf _{n \rightarrow \infty} n \cdot\|n \theta\|=\liminf _{n \rightarrow \infty} n \cdot\left|T_{\theta}^{n}(x)-x\right| \geq c,
$$

where $\|\cdot\|$ denotes the distance to its nearest integer and $T_{\theta}$ is the rotation by $\theta$ on the unit circle. In this paper, we investigate the bounded type condition for the interval exchange map.

An interval exchange map (i.e.m.) is determined by combinatorial data and length data. The combinatorial data consists of a finite set $\mathcal{A}$ for the subintervals and of two bijections $\left(\pi_{t}, \pi_{b}\right)$ from $\mathcal{A}$ onto $\{1, \ldots, d\}(|\mathcal{A}|=d)$ : these indicate in which order the intervals are met before and after the map. The length data $\left(\lambda_{\alpha}\right)_{\alpha \in \mathcal{A}}$ give the length $\lambda_{\alpha}>0$ of the corresponding interval.

2000 Mathematics Subject Classification. 37E05, 11J70.

Key words and phrases. interval exchange map, bounded type, recurrence time, Rauzy-Veech induction.

This work was partially supported by the National Research Foundation of Korea(NRF) (2012R1A1A2004473). 
We set

$$
p_{\alpha}:=\sum_{\pi_{t}(\beta)<\pi_{t}(\alpha)} \lambda_{\beta}, \quad q_{\alpha}:=\sum_{\pi_{b}(\beta)<\pi_{b}(\alpha)} \lambda_{\beta}, \quad \lambda^{*}=\sum_{\alpha} \lambda_{\alpha} .
$$

The i.e.m. $T$ associated to these data is defined as

$$
T(x)=x-p_{\alpha}+q_{\alpha} \text { for } x \in I_{\alpha}:=\left[p_{\alpha}, p_{\alpha}+\lambda_{\alpha}\right) .
$$

In the following, we will consider only combinatorial data $\left(\mathcal{A}, \pi_{t}, \pi_{b}\right)$ which are admissible, i.e., $\pi_{t}^{-1}(\{1, \ldots, k\}) \neq \pi_{b}^{-1}(\{1, \ldots, k\})$ for $1 \leq k<d$. Moreover, we will assume our maps to have the Keane property: if there exist $\alpha, \beta \in \mathcal{A}$ and integer $m$ such that $T^{m}\left(p_{\alpha}\right)=p_{\beta}$ and $\pi_{t}(\beta)>1$. The Keane property is the appropriate notion of irrationality for i.e.m. [5].

For admissible interval exchange maps with the Keane property we can introduce the generalization of continued fractions to i.e.m.'s (see [14, 15, for a more detailed discussion) due to the work of Rauzy [11, Veech [12] and Zorich [16, 17].

We say that $T$ is of top type (respectively bottom type) if one has $\lambda_{\alpha_{t}} \geq \lambda_{\alpha_{b}}$ (respectively $\lambda_{\alpha_{b}} \geq \lambda_{\alpha_{t}}$ ); we then define a new i.e.m. $\mathcal{V}(T)$ as the induced map on $\left[0, \lambda^{*}-\lambda_{\alpha_{b}}\right)$ (respectively $\left[0, \lambda^{*}-\lambda_{\alpha_{t}}\right)$ ), which is given by a new admissible pair $\mathcal{R}_{t}\left(\pi_{t}, \pi_{b}\right)$ and the lengths $\left(\hat{\lambda}_{\alpha}\right)_{\alpha \in \mathcal{A}}$ given by

$$
\begin{cases}\hat{\lambda}_{\alpha}=\lambda_{\alpha} & \text { if } \alpha \neq \alpha_{t}, \\ \hat{\lambda}_{\alpha_{t}}=\lambda_{\alpha_{t}}-\lambda_{\alpha_{b}} & \text { otherwise }\end{cases}
$$

for the top type $T$; a new admissible pair $\mathcal{R}_{b}\left(\pi_{t}, \pi_{b}\right)$ and the lengths $\left(\hat{\lambda}_{\alpha}\right)_{\alpha \in \mathcal{A}}$ given by

$$
\begin{cases}\hat{\lambda}_{\alpha}=\lambda_{\alpha} & \text { if } \alpha \neq \alpha_{b}, \\ \hat{\lambda}_{\alpha_{b}}=\lambda_{\alpha_{b}}-\lambda_{\alpha_{t}} & \text { otherwise }\end{cases}
$$

for the bottom type $T$.

The Rauzy diagram is the graph of vertices obtained by saturation of $\left(\pi_{t}, \pi_{b}\right)$ under the action of $\mathcal{R}_{t}$ and $\mathcal{R}_{b}$ and two arrows joining $\left(\pi_{t}, \pi_{b}\right)$ to $\mathcal{R}_{t}\left(\pi_{t}, \pi_{b}\right), \mathcal{R}_{b}\left(\pi_{t}, \pi_{b}\right)$. For an arrow joining $\left(\pi_{t}, \pi_{b}\right)$ to $\mathcal{R}_{t}\left(\pi_{t}, \pi_{b}\right)$ (respectively $\mathcal{R}_{b}\left(\pi_{t}, \pi_{b}\right)$ ) the element $\alpha_{t} \in \mathcal{A}$ (respectively $\alpha_{b} \in \mathcal{A}$ ) is called the winner and the element $\alpha_{b} \in \mathcal{A}$ (respectively $\alpha_{t} \in \mathcal{A}$ ) is called the loser.

Iterating this process, we obtain a sequence of i.e.m. $T(n)=\mathcal{V}^{n}(T)$ and an infinite path in the Rauzy diagram starting from $\left(\pi_{t}, \pi_{b}\right)$. In fact, a further property 
of irrational interval exchange maps (i.e. with the Keane property) is that every letter is taken infinitely many times as the winner in the infinite path (in the Rauzy diagram) associated to $T$. This property is fundamental in order to be able to group together several iterations of $\mathcal{V}$ to obtain the accelerated Zorich continued fraction algorithm.

For an arrow $\gamma$ with winner $\alpha$ and loser $\beta$ in the Rauzy diagram let $B_{\gamma}=\mathbb{I}+E_{\beta \alpha}$ where $\mathbb{I}$ is the identity matrix and $E_{\beta \alpha}$ is the matrix with only non-zero entry 1 at $(\beta, \alpha)$. For a finite path $\underline{\gamma}=\gamma_{1} \gamma_{2} \cdots \gamma_{n}$ in the Rauzy diagram we associate a $S L\left(\mathbb{Z}^{\mathcal{A}}\right)$ matrix with non-negative entries

$$
B_{\underline{\gamma}}=B_{\gamma_{n}} \cdots B_{\gamma_{1}} .
$$

Let $\gamma^{T}(m, n)=\gamma(m, n), m \leq n$ be the path in the Rauzy diagram from the permutation of $T(m)$ to the permutation of $T(n)$ and denote

$$
B(m, n)=B_{\gamma(m, n)}, \quad B(n)=B(0, n) .
$$

Let $\lambda(n)$ be the length data of $T(n)$. Then we have

$$
\lambda(m)=\lambda(n) B(m, n) .
$$

Zorich's accelerated continued fraction algorithm is obtained by considering by $\left(\mathcal{V}^{n_{k}}\right)_{k \geq 0}$ where $n_{k}$ is the following sequence: $n_{0}=0$ and $n_{k+1}>n_{k}$ is chosen so as to assure that $\gamma\left(n_{k}, n_{k+1}\right)$ is the longest path whose arrows have the same winner.

A further acceleration algorithm by Marmi-Moussa-Yoccoz [9] is obtained by $\left(\mathcal{V}^{m_{k}}\right)_{k \geq 0}$ where $m_{k}$ is defined as follows: $m_{0}=0$ and $m_{k+1}>m_{k}$ is the largest integer such that all letters in $\mathcal{A}$ are taken as winner by arrows in $\gamma\left(m_{k}, m_{k+1}\right)$.

As the irrational rotations, bounded type interval exchange map $T$ can be characterized by its continued fraction matrix.

(A) The MMY cocycle matrices is bounded, i.e.,

$$
\left\|B\left(m_{k}, m_{k+1}\right)\right\| \leq M
$$

(Z) The Zorich cocycle matrices are bounded, i.e.,

$$
\left\|B\left(n_{k}, n_{k+1}\right)\right\| \leq M .
$$

Let $\Delta(T)$ be the minimal distance between discontinuities of $T$. We have the following characterization of the bounded type i.e.m. by dynamics of $T$. 
(D) There is a constant $c$ such that

$$
\Delta\left(T^{n}\right) \geq \frac{c}{n} \text { for all } n .
$$

(U) There is a constant $c>0$ such that for all $x$

$$
\liminf _{n \rightarrow \infty} n \cdot\left|T^{n}(x)-x\right| \geq c .
$$

Here and after, the matrix norm is $\|A\|=\sum_{i, j}\left|a_{i j}\right|$ for a matrix $A=\left(a_{i, j}\right)$.

Main Theorem. (i) (A) bounded type and (D) bounded type are equivalent.

(ii) (D) bounded type implies (U) bounded type.

(iii) (U) bounded type implies $(Z)$ bounded type.

The inverse of (ii) and (iii) do not hold. See Section 4 for the examples. The proof of (i) is given in Section 2 and In Section 3, the proofs of (ii) and (iii) are presented.

In the last ten years, there has been progress in diophanitne condition of the i.e.m. (see [2, 3, 8]) and the Roth type diophantine condition for the i.e.m. has been studied in [6, 7, 10]. Condition (D) is considered by Boshernitzan for unique ergodicity [1]. Condition (D) and the bounded minimum saddle connection are equivalent, see [4, 13].

While we were writing this paper, we found out that the equivalence (i) in the Main Theorem is also proved in the recent preprint [4 by Hubert, Marchese and Ulcigrai. In [4 the authors consider a related acceleration of the algorithm (the positive acceleration), whose matrices are uniformly bounded if and only if (A) holds and show that bounded positive matrices is equivalent to (D) (see [4, Corollary 4.8 combined with Proposition 1.1). From their proof one can also obtain quantitative relations between the constant $c$ in (D) and the norm of the matrices (see Theorem 4.7 in (4), but our proof is much shorter and less combinatorially involved than theirs (compare with Appendix C in [4]).

\section{The Bounded MMY COCYClE CONDITION AND THE BOUNDED GAP CONDITION OF DISCONTINUITIES}

In this section, we prove (i) of the main theorem through Proposition 2.2 and 2.5. Let $r=\max (2 d-3,2)$. By [9], each entry of the matrix $B\left(m_{k}, m_{k+r}\right)$ is strictly positive. 
Assume that $\left\|B\left(m_{k}, m_{k+1}\right)\right\| \leq M$ for all $k$. Then clearly we have

$$
\left\|B\left(m_{k}, m_{k+r}\right)\right\| \leq M^{r}, \quad\left\|B\left(m_{k+r}\right)\right\| \leq M^{r}\left\|B\left(m_{k}\right)\right\| .
$$

Since

$$
\sum_{\alpha \in \mathcal{A}} \lambda_{\alpha}\left(m_{k+r}\right) B_{\alpha \beta}\left(m_{k}, m_{k+r}\right)=\lambda_{\beta}\left(m_{k}\right)
$$

and $B_{\alpha \beta}\left(m_{k}, m_{k+r}\right) \geq 1$ for all $\alpha, \beta \in \mathcal{A}$, we have

$$
\min _{\alpha \in \mathcal{A}} \lambda_{\alpha}\left(m_{k}\right)>\max _{\alpha \in \mathcal{A}} \lambda_{\alpha}\left(m_{k+r}\right)
$$

Also we have

$$
\sum_{\alpha, \beta \in \mathcal{A}} \lambda_{\alpha}\left(m_{k}\right) B_{\alpha \beta}\left(m_{k}\right)=1
$$

which follows

$$
\left\|B\left(m_{k}\right)\right\| \cdot \max _{\alpha \in \mathcal{A}} \lambda_{\alpha}\left(m_{k}\right) \geq 1 .
$$

Let $B_{\alpha}\left(m_{k}\right)=\sum_{\beta} B_{\alpha \beta}\left(m_{k}\right)$. Then for any $\alpha \in \mathcal{A}$ we have

$$
\left\|B\left(m_{k}\right)\right\|=\sum_{\beta, \delta} B_{\beta \delta}\left(m_{k}\right) \leq \sum_{\beta, \delta} B_{\alpha \delta}\left(m_{k}, m_{k+r}\right) B_{\delta \beta}\left(m_{k}\right)=B_{\alpha}\left(m_{k+r}\right) .
$$

Lemma 2.1 ([], Lemma 4.2 and 4.3). If $0<n \leq \min _{\alpha} B_{\alpha}\left(m_{k}\right)$, then

$$
\min _{\alpha \in \mathcal{A}} \lambda_{\alpha}\left(m_{k+r}\right) \leq \Delta\left(T\left(m_{k}\right)^{2}\right) \leq \Delta\left(T^{n}\right) .
$$

For any $\min _{\alpha} B_{\alpha}\left(m_{k-1}\right)<n \leq \min _{\alpha} B_{\alpha}\left(m_{k}\right)$ we have

$$
\begin{aligned}
& \Delta\left(T^{n}\right) \geq \min _{\alpha} \lambda_{\alpha}\left(m_{k+r}\right), \quad \text { by Lemma } 2.1 \\
& \geq \max \lambda_{\alpha}\left(m_{k+2 r}\right), \quad \text { by (3), } \\
& \geq \frac{1}{\left\|B\left(m_{k+2 r}\right)\right\|}, \quad \text { by (4), } \\
& \geq \frac{1}{M^{4 r}} \cdot \frac{1}{\left\|B\left(m_{k-2 r}\right)\right\|}, \quad \text { by (2), } \\
& >\frac{1}{M^{4 r}} \cdot \frac{1}{\min _{\alpha} B_{\alpha}\left(m_{k-r}\right)}, \quad \text { by (5) } \\
& >\frac{1}{M^{4 r}} \cdot \frac{1}{n} \quad \text { by the assumption. }
\end{aligned}
$$

Therefore, we have the following: 
Proposition 2.2. If the $M M Y$ cocycle of $T$ satisfies $\left\|B\left(m_{k}, m_{k+1}\right)\right\| \leq M$, then we have

$$
\Delta\left(T^{n}\right)>\frac{1}{M^{4 r}} \cdot \frac{1}{n}
$$

Lemma 2.3. We have either

$$
\min _{\alpha} \lambda_{\alpha}\left(m_{k}\right) \cdot \sqrt{\left\|B\left(m_{k}, m_{k+1}\right)\right\|}<\lambda^{*}\left(m_{k}\right)
$$

or

$$
\min _{\alpha} \lambda_{\alpha}\left(m_{k+1}\right) \cdot \sqrt{\left\|B\left(m_{k}, m_{k+1}\right)\right\|}<\lambda^{*}\left(m_{k+1}\right)
$$

Proof. For each $k$ let $\alpha=\alpha(k) \in \mathcal{A}$, depending on $k$, be the letter which is not taken as the winner of the arrows in the path $\gamma\left(m_{k}, m_{k+1}\right)$. Then

$$
\lambda_{\alpha}\left(m_{k}\right)=\lambda_{\alpha}\left(m_{k+1}\right) .
$$

Now we have two cases:

Case (i) : $\lambda_{\alpha}\left(m_{k}\right) \cdot \sqrt{\left\|B\left(m_{k}, m_{k+1}\right)\right\|}<\lambda^{*}\left(m_{k}\right)$, which implies the lemma.

Case (ii) : $\lambda_{\alpha}\left(m_{k}\right) \cdot \sqrt{\left\|B\left(m_{k}, m_{k+1}\right)\right\|} \geq \lambda^{*}\left(m_{k}\right)$

Since

$$
\sum_{\alpha, \beta \in \mathcal{A}} \lambda_{\alpha}\left(m_{k+1}\right) B_{\alpha \beta}\left(m_{k}, m_{k+1}\right)=\lambda^{*}\left(m_{k}\right)
$$

we have

$$
\min _{\alpha} \lambda_{\alpha}\left(m_{k+1}\right) \cdot\left\|B\left(m_{k}, m_{k+1}\right)\right\|<\lambda^{*}\left(m_{k}\right)<\max _{\alpha} \lambda_{\alpha}\left(m_{k+1}\right) \cdot\left\|B\left(m_{k}, m_{k+1}\right)\right\| .
$$

Thus, there exists $\beta \in \mathcal{A}$ such that

$$
\lambda_{\beta}\left(m_{k+1}\right) \cdot\left\|B\left(m_{k}, m_{k+1}\right)\right\|<\lambda^{*}\left(m_{k}\right) \leq \lambda_{\alpha}\left(m_{k}\right) \cdot \sqrt{\left\|B\left(m_{k}, m_{k+1}\right)\right\|} .
$$

Therefore, we have

$$
\lambda^{*}\left(m_{k+1}\right)>\lambda_{\alpha}\left(m_{k+1}\right)=\lambda_{\alpha}\left(m_{k}\right)>\lambda_{\beta}\left(m_{k+1}\right) \cdot \sqrt{\left\|B\left(m_{k}, m_{k+1}\right)\right\|} .
$$

Lemma 2.4. Let $\alpha \in \mathcal{A}$ be the winner of $\gamma(n-1, n)$ and the loser of $\gamma(n, n+1)$. For large $n$, if $\lambda_{\alpha}(n)<\lambda^{*}(n) / M^{d}, M>d$, then there is an integer $s, 1 \leq s<d$, such that

$$
\Delta\left(T^{\left\lfloor 2 M^{s} / \lambda^{*}(n)\right\rfloor}\right)<(d-1) \frac{\lambda^{*}(n)}{M^{s+1}} .
$$


Proof. Let for $0 \leq i<d$

$$
\mathcal{A}_{i}=\left\{\beta \in \mathcal{A}: \frac{\lambda^{*}(n)}{M^{i+1}} \leq \lambda_{\beta}(n)<\frac{\lambda^{*}(n)}{M^{i}}\right\}
$$

and

$$
\mathcal{A}_{d}=\left\{\beta \in \mathcal{A}: \lambda_{\beta}(n)<\frac{\lambda^{*}(n)}{M^{d}}\right\} .
$$

Then, by the assumption, $\alpha \in \mathcal{A}_{d} \neq \emptyset$. Since there is an $\beta \in \mathcal{A}$ such that $\lambda_{\beta}(n)>$ $\lambda^{*}(n) / d>\lambda^{*}(n) / M$, neither $\mathcal{A}_{0}$ is an empty set.

Since there are $d$ elements in $\mathcal{A}$, there exist an $s, 1 \leq s<d$, such that $\mathcal{A}_{s}$ is empty. Let

$$
\mathcal{A}_{\text {big }}=\bigcup_{i=0}^{s-1} \mathcal{A}_{i}, \quad \mathcal{A}_{\text {small }}=\bigcup_{i=s+1}^{d} \mathcal{A}_{i} .
$$

Both $\mathcal{A}_{\text {big }}$ and $\mathcal{A}_{\text {small }}$ are nonempty.

Take $m$ with $m<n$ be the smallest integer as no loser in $\gamma(m+1, n)$ belongs to $\mathcal{A}_{\text {big. }}$. Put $\mu \in \mathcal{A}_{\text {big }}$ as the loser of the arrow $\gamma(m, m+1)$. Let $\nu$ be the winner of the arrow $\gamma(m, m+1)$. Then $\nu \in \mathcal{A}_{\text {small }}$. (if $\nu \in \mathcal{A}_{\text {big }}$, then $\nu \neq \alpha$ and $\nu$ should be a loser in $\gamma(m+1, n))$

Hence we have $\lambda_{\nu}(m+1)=\lambda_{\nu}(m)-\lambda_{\mu}(m)$ and

$$
\begin{aligned}
& B_{\nu}(m+1)=B_{\nu}(m)<\frac{1}{\lambda_{\nu}(m)}<\frac{1}{\lambda_{\mu}(m)} \leq \frac{M^{s}}{\lambda^{*}(n)}, \\
& B_{\mu}(m+1)=B_{\nu}(m)+B_{\mu}(m)<\frac{1}{\lambda_{\nu}(m)}+\frac{1}{\lambda_{\mu}(m)}<\frac{2}{\lambda_{\mu}(m)} \leq \frac{2 M^{s}}{\lambda^{*}(n)} .
\end{aligned}
$$

There are two cases:

(i) $\pi_{t}^{(m)}(\mu)=d$ and $\pi_{b}^{(m)}(\nu)=d$ :

Then we have $\pi_{t}^{(m+1)}(\nu)=\pi_{t}^{(m)}(\nu)<d$ and $\pi_{t}^{(m+1)}(\mu)=\pi_{t}^{(m)}(\nu)+1$.

Since no letter in $\mathcal{A}_{\text {big }}$ is taken as the winner or the loser of the arrows of $\gamma(m+1, n)$,

$$
I_{\nu}(m)=I_{\nu}(m+1) \cup I_{\mu}(m+1) \subset\left[0, \lambda^{*}(n)\right)
$$

and

$$
I_{\nu}(m+1)=\left[p_{\nu}(m+1), p_{\mu}(m+1)\right) .
$$

Since $p_{\nu}(m+1), p_{\mu}(m+1)$ are discontinuity points of $T(n)$ and $\pi_{b}^{(m+1)}(\nu)=d$, $m+1 \leq n$, we have

$$
I_{\nu}(m+1)=\bigsqcup_{\beta \in \mathcal{A}^{\prime}} I_{\beta}(n) \text { for some } \mathcal{A}^{\prime} \subset A_{\text {small }} .
$$


Therefore, we have

$$
p_{\mu}(m+1)-p_{\nu}(m+1)=\lambda_{\nu}(m+1)<\left|\mathcal{A}_{\text {small }}\right| \cdot \frac{\lambda^{*}(n)}{M^{s+1}} \leq(d-1) \frac{\lambda^{*}(n)}{M^{s+1}} .
$$

Since

$$
\begin{aligned}
& p_{\mu}(m+1) \in D\left(T^{B_{\mu}(m+1)}\right)=D\left(T^{B_{\nu}(m)+B_{\mu}(m)}\right) . \\
& p_{\nu}(m+1) \in D\left(T^{B_{\nu}(m+1)}\right)=D\left(T^{B_{\nu}(m)}\right)
\end{aligned}
$$

we have

$$
p_{\mu}(m+1)-p_{\nu}(m+1) \geq \Delta\left(T^{B_{\mu}(m+1)}\right) .
$$

(ii) $\pi_{t}^{(m)}(\nu)=d$ and $\pi_{b}^{(m)}(\mu)=d$ :

Then we have $\pi_{b}^{(m+1)}(\nu)=\pi_{b}^{(m)}(\nu)<d$ and $\pi_{b}^{(m+1)}(\mu)=\pi_{b}^{(m)}(\nu)+1$. Similarly with case (i), we have

$$
q_{\mu}(m+1)-q_{\nu}(m+1)=\lambda_{\nu}(m+1)<(d-1) \frac{\lambda^{*}(n)}{M^{s+1}} .
$$

Since

$$
\begin{aligned}
& q_{\mu}(m+1) \in D\left(T^{-B_{\mu}(m+1)}\right)=D\left(T^{-B_{\nu}(m)-B_{\mu}(m)}\right), \\
& q_{\nu}(m+1) \in D\left(T^{-B_{\nu}(m+1)}\right)=D\left(T^{-B_{\nu}(m)}\right)
\end{aligned}
$$

we have

$$
q_{\mu}(m+1)-q_{\nu}(m+1) \geq \Delta\left(T^{-B_{\mu}(m+1)}\right)=\Delta\left(T^{B_{\mu}(m+1)}\right) .
$$

Note that $\Delta(T)=\Delta\left(T^{-1}\right)$.

Proposition 2.5. If $\lim \sup _{k \rightarrow \infty}\left\|B\left(m_{k}, m_{k+1}\right)\right\|=\infty$, then $\liminf \inf _{n \rightarrow \infty} n \cdot \Delta\left(T^{n}\right)=$ 0.

Proof. For any give big $M>0$, by Lemma 2.3, there are infinitely many $k$ and $\alpha$ (depending on $k$ ) satisfying

$$
\lambda_{\alpha}\left(m_{k}\right)=\min _{\beta \in \mathcal{A}} \lambda_{\beta}\left(m_{k}\right)<\frac{\lambda^{*}\left(m_{k}\right)}{M} .
$$

Let $\ell_{k}(\alpha)=\max \left\{n \leq m_{k}: \alpha\right.$ is the winner of $\left.\gamma(n-1, n)\right\}$. Since $\lambda_{\alpha}\left(m_{k}\right)=$ $\min _{\beta \in \mathcal{A}} \lambda_{\beta}\left(m_{k}\right), \alpha$ cannot be the winner of $\gamma\left(m_{k}, m_{k}+1\right)$. Thus $\alpha$ should be the winner of an arrow in $\gamma\left(m_{k-1}, m_{k}\right)$, which yields

$$
m_{k-1}<\ell_{k}(\alpha)<m_{k} .
$$


Thus,

$$
\lambda_{\alpha}\left(\ell_{k}(\alpha)\right)=\lambda_{\alpha}\left(m_{k}\right)<\frac{\lambda^{*}\left(m_{k}\right)}{M} \leq \frac{\lambda^{*}\left(\ell_{k}(\alpha)\right)}{M} .
$$

Hence, we can choose infinitely many n's satisfying the condition for Lemma 2.4 which completes the proof.

\section{Bounded Zorich COCYCle CONDition AND THE Uniform RECURRENCE}

\section{CONDITION}

In this section, we prove (ii) and (iii) of the main theorem.

The proof of (ii) and (iii) in the main theorem are directly obtained by the following propositions:

Proposition 3.1. If $n \cdot\left|T^{n}(x)-x\right|<c$ for some $x$, then we have

$$
\Delta\left(T^{2 n}\right)<\frac{c}{n}
$$

Proof. Let $[a, b)$ be the maximal interval containing $x$ on which $T^{n}$ is continuous. If $b-a<c / n$, then the proof is completed.

Suppose that $b-a \geq \frac{c}{n}$. Let $\delta=T^{n}(x)-x$. Then $|\delta|<\frac{c}{n} \leq b-a$ and $T^{n}[a, b)=[a+\delta, b+\delta)$. Therefore, either $T^{n}(a-\delta)=a$ or $T^{n}(b-\delta)=b$ is a discontinuous point of $T^{n}$ depending on $\delta<0$ or $\delta>0$. Hence, either $a-\delta$ or $b-\delta$ is a discontinuous point of $T^{2 n}$, which implies that

$$
\Delta\left(T^{2 n}\right) \leq|\delta|<\frac{c}{n}
$$

since $a$ and $b$ are discontinuous point of $T^{n}$ or the end point of the interval.

Proposition 3.2. If $n_{k+1}-n_{k} \geq d-1$ and $\left\|B\left(n_{k}, n_{k+1}\right)\right\|>2 d$, then there exist $x$ and $m$ such that

$$
m \cdot\left|T^{m}(x)-x\right|<\frac{d}{\left\|B\left(n_{k}, n_{k+1}\right)\right\|-2 d}
$$

Proof. Let $\alpha \in \mathcal{A}$ be the winner of the arrows and $\mathcal{A}^{\prime}$ be the set of the losers of the arrows in the path $\gamma\left(n_{k}, n_{k+1}\right)$. If $\pi_{t}^{\left(n_{k}\right)}(\alpha)=d$, then $\mathcal{A}^{\prime}=\left\{\beta \in \mathcal{A}: \pi_{b}^{\left(n_{k}\right)}(\beta)>\right.$ $\left.\pi_{b}^{\left(n_{k}\right)}(\alpha)\right\}$ and $\pi_{b}^{(n)}$ is the cyclic permutation on $\mathcal{A}^{\prime}$ for $n_{k} \leq n \leq n_{k+1}$. For each $\beta \in \mathcal{A}^{\prime}$ put $h_{\beta}=B_{\beta \alpha}\left(n_{k}, n_{k+1}\right)$, the number of arrows, of which loser is $\beta \in \mathcal{A}^{\prime}$, in the path $\gamma\left(n_{k}, n_{k+1}\right)$. Put

$$
h:=\left\lfloor\frac{n_{k+1}-n_{k}}{\left|\mathcal{A}^{\prime}\right|}\right\rfloor \geq 1 .
$$


Then $h \leq h_{\beta} \leq h+1$ for all $\beta \in \mathcal{A}^{\prime}$ and

$$
\left\|B\left(n_{k}, n_{k+1}\right)\right\|=d+n_{k+1}-n_{k} \leq d+(h+1) \cdot\left|\mathcal{A}^{\prime}\right|<d(h+2) .
$$

Let

$$
\frac{\lambda_{\alpha}\left(n_{k}\right)}{h}>\frac{\lambda_{\alpha}\left(n_{k}\right)-\lambda_{\alpha}\left(n_{k+1}\right)}{h}=\frac{\sum_{\beta \in \mathcal{A}^{\prime}} h_{\beta} \lambda_{\beta}\left(n_{k}\right)}{h} \geq \sum_{\beta \in \mathcal{A}^{\prime}} \lambda_{\beta}\left(n_{k}\right) .
$$

Let $m=B_{\alpha}\left(n_{k}\right)$. Then on $x \in I_{\alpha}\left(n_{k}\right)$ we have

$$
\left|T^{m}(x)-x\right|=\sum_{\beta \in \mathcal{A}^{\prime}} \lambda_{\beta}\left(n_{k}\right)<\frac{\lambda_{\alpha}\left(n_{k}\right)}{h} .
$$

Hence we have for $x \in I_{\alpha}\left(n_{k}\right)$

$m \cdot\left|T^{m}(x)-x\right|<B_{\alpha}\left(n_{k}\right) \cdot \frac{\lambda_{\alpha}\left(n_{k}\right)}{h}<\frac{B_{\alpha}\left(n_{k}\right) \lambda_{\alpha}\left(n_{k}\right)}{\left\|B\left(n_{k}, n_{k+1}\right)\right\| / d-2}<\frac{1}{\left\|B\left(n_{k}, n_{k+1}\right)\right\| / d-2}$.

For the case $\pi_{b}^{\left(n_{k}\right)}(\alpha)=d$ we have the same procedure.

\section{EXAmples}

The example satisfying condition (U) without (D) can be obtained by 3 -interval exchanges. Let $T$ be a 3 interval exchange map with permutation $\left(\begin{array}{ccc}A & B \\ B & A\end{array}\right)$ and the length data $\left(\lambda_{A}, \lambda_{B}, \lambda_{C}\right)$. Then $T$ is the induced transformation of the translation

$$
\bar{T}(x)= \begin{cases}x+\lambda_{B}+\lambda_{C}, & x+\lambda_{B}+\lambda_{C}<1+\lambda_{B}, \\ x-\lambda_{A}-\lambda_{B}, & x+\lambda_{B}+\lambda_{C} \geq 1+\lambda_{B}\end{cases}
$$

on the unit interval $[0,1)$. Then condition $(\mathrm{U})$ or $(\mathrm{Z})$ are equivalent that $\bar{T}$ is of bounded type.

Let $T$ has the infinite path in the Rauzy diagram given by sequence of the winners of the arrows as follows

$$
A B A\left(A^{2} C^{2}\right)^{n_{1}} A B A\left(A^{2} C^{2}\right)^{n_{2}} A B A\left(A^{2} C^{2}\right)^{n_{3}} \cdots
$$

with $n_{k} \rightarrow \infty$. Clearly, $T$ does not satisfies condition $(A)$. However, the infinite path in the Rauzy diagram for $\bar{T}$ is

\section{$\bar{A} \bar{C} \bar{A} \bar{C} \bar{A} \bar{C} \bar{A} \bar{C} \cdots$,}

if we consider $\bar{T}$ as the 2-interval exchange map with permutation $\left(\begin{array}{c}\bar{A} \\ \bar{C}\end{array} \bar{A}\right)$ and the length data $\left(\lambda_{\bar{A}}, \lambda_{\bar{C}}\right)=\left(\lambda_{A}+\lambda_{B}, \lambda_{C}+\lambda_{B}\right)$. Therefore, $\bar{T}$ is the rotation by the golden mean $g^{-1}=(\sqrt{5}-1) / 2$. It follows that $T$ satisfies condition $(U)$. 
An example satisfying condition $(\mathrm{Z})$ but not condition $(\mathrm{U})$ is explained in [6]: Let $T$ be the 4-interval exchange map with permutation data $\left(\begin{array}{llll}A & B & D & C \\ D & A & C & B\end{array}\right)$ with infinite path in the Rauzy diagram

$$
C B^{3}\left(D^{2} A^{3} D\right)^{2^{1}} B \cdot C B^{3}\left(D^{2} A^{3} D\right)^{2^{2}} B \cdots C B^{3}\left(D^{2} A^{3} D\right)^{2^{k}} B \cdots
$$

Then $T$ satisfies condition $(\mathrm{Z})$ since there is no long sequence of the same winner.

However, it was shown in [6, Section 9] that for $x \in I_{C}(n), n=\sum_{i=1}^{k}\left(5+6 \cdot 2^{i}\right)+3=$ $2 k+12\left(2^{k}-1\right)+3$,

$$
n \cdot\left|T^{n}(x)-x\right|<\frac{2 g^{2^{k+2}+5 k}}{g^{2^{k+3}+k-4}}
$$

where $g=\frac{1}{2}(\sqrt{5}+1)$.

\section{ACKNOWLEDGEMENT}

The authors wish to thank Corinna Ulcigrai and Luca Marchese for introducing their works and very helpful comments.

\section{REFERENCES}

[1] M. Boshernitzan, A condition for minimal exchange maps to be a uniquely ergodic, Duke J. Math. 52 (1985), 723-752.

[2] M. Boshernitzan and J. Chaika, Diophantine properties of IETs and general systems: quantitative proximality and connectivity, Invent. Math. 192 (2013), 375-412.

[3] J. Chaika, Shrinking targets for IETs: extending a theorem of Kurzweil. Geom. Funct. Anal. 21 (2011), 1020-1042.

[4] P. Hubert, L. Marchese and C. Ulcigrai, Lagrange Spectra in Teichmüller Dynamics via renormalization, arXiv:1209.0183

[5] M. Keane, Interval exchange transformations, Math. Z. 141 (1975), 25-31.

[6] D.H. Kim, Diophantine type of interval exchange maps, Ergod. Th. Dynam. Sys., to appear.

[7] D.H. Kim and S. Marmi, The recurrence time for interval exchange maps, Nonlinearity 21 (2008), 2201-2210.

[8] L. Marchese, The Khinchin theorem for interval-exchange transformations J. Mod. Dyn. 5 (2011), 123-183.

[9] S. Marmi, P. Moussa and J.-C. Yoccoz, The cohomological equation for Roth type interval exchange maps, J. Amer. Math. Soc. 18 (2005), 823-872.

[10] S. Marmi, P. Moussa and J.-C. Yoccoz, Linearization of generalized interval exchange maps, Ann. Math. 176 (2012), 1583-1646.

[11] G. Rauzy, Échanges d'intervalles et transformations induites, Acta Arith. 34 (1979), 315-328 
[12] W. Veech, Gauss meuasures for transformations on the space of interval exchange maps, Ann. Math. 115 (1982), 201-242.

[13] Y. Vorobets, Plane structures and billiards in rational polygons: the Veech alternative (Russian), Uspekhi Mat. Nauk 51 (1996), 3-42; translation in Russian Math. Surveys 51 (1996), 779817.

[14] J.-C. Yoccoz, Continued fraction algorithms for interval exchange maps: an introduction Frontiers in number theory, physics, and geometry. I, 401-435, Springer, Berlin, 2006.

[15] J.-C. Yoccoz, Interval exchange maps and translation surfaces, Proceeding of Clay Mathematics Institute workshop, AMS 2008.

[16] A. Zorich, Finite Gauss measure on the space of interval exchange transformations. Lyapunov exponents Annales de l'Institut Fourier 46 fasc. 2 (1996), 325-370

[17] A. Zorich Deviation for interval exchange transformations Ergod. Th. Dyn. Sys. 17 (1997), $1477-1499$

Department of Mathematics Education, Dongguk University-Seoul, Seoul 100-715, Korea.

E-mail address: kim2010@dongguk.edu

Scuola Normale Superiore, Piazza dei Cavalieri 7, 56126 Pisa, Italy

E-mail address: s.marmi@sns.it 